\title{
Grover's search algorithm: An optical approach
}

\author{
P. G. Kwiat, J. R. Mitchell, P. D. D. Schwindt, and A. G. White \\ Physics Division, P-23, Los Alamos National Laboratory, Los Alamos, New Mexico 87545 \\ (To appear in a special issue of the Journal of Modern Optics-"The Physics of Quantum Information")
}

\begin{abstract}
The essential operations of a quantum computer can be accomplished using solely optical elements, with different polarization or spatial modes representing the individual qubits. We present a simple all-optical implementation of Grover's algorithm for efficient searching, in which a database of four elements is searched with a single query. By 'compiling' the actual setup, we have reduced the required number of optical elements from 24 to only 12 . We discuss the extension to larger databases, and the limitations of these techniques.
\end{abstract}

\section{Introduction}

It is now well-known that quantum computation, if implementable, could enable tremendous improvements over traditional computing methods for certain types of problems, such as factoring large numbers into primes [1], and efficiently searching a database [2]. Thus far, the implementation of actual algorithms has been limited to schemes employing bulk NMR methods [3, 4 , 1 , although basic gate operations have also been performed using cooled ions [5] and also cavity QED methods [6]. To date there have been a number of discussions on the implementation of basic quantum operations using purely optical methods [7] 11]. The central idea is that individual qubits can be represented by different polarization or spatial-mode degrees of freedom. The difference from a genuine quantum computer with distinct entangleable registers is that the optical implementation requires a number of elements which grows exponentially with the number of qubits. Nevertheless, as we shall see, it is possible to 'compile' the circuits, allowing one to easily realize and test nontrivial algorithms involving several bits. One conclusion of this approach is that a quantum computer is essentially a (complicated) interferometer [12].

As shown in 10], with this representation all the basic circuit elements of quantum computation can be accomplished using only linear passive optical elements. This includes the Controlled Not (CNOT) gate, which entangles different bits, and the simpler Walsh-Hadamard $(\mathrm{WH})$ transform: $0 \rightarrow(0+1) / \sqrt{2}$ and $1 \rightarrow(0-1) / \sqrt{2}$. For the present work we shall only need the latter, in addition to the ability to apply various phase shifts on the bits. If one considers a qubit based on the polarization degree-of-freedom, where horizontal $(\mathrm{H})$ and vertical $(\mathrm{V})$ polarization represent 0 and 1 , respectively, then the $\mathrm{WH}$ transformation can be accomplished with a half waveplate (HWP) oriented at $22.5^{\circ}$ to the horizontal. Similarly, a simple 50-50 beamsplitter (BS) performs the WH transform on a right-propagating spatial mode $(\equiv 0)$ and an upward-propagating spatial mode ( $\equiv 1)$, after two extra phase shifters are included 1. We will now describe how a nontrivial quantum circuit - Grover's algorithm for efficiently searching a database - may be constructed using these elements.

\section{Grover's Search Algorithm}

\subsection{General Description}

Consider a database of $N$ elements (e.g., a list of integers) exactly one of which is 'marked' as satisfying some desirable characteristic (e.g., is a prime number). Typically, one would expect to have to look at and test roughly half the database (making $\sim N / 2$ 'queries' on average) before locating this element. Grover's algorithm uses the parallelism afforded by quantum superposition to accomplish the task with only $\sim \sqrt{N}$ queries. Since it is described in detail elsewhere [2, 14,13], here we present without proof the necessary steps. Enough qubits are input to the computer to encode the elements of the database $-n$ qubits suffice for a $2^{n}$-element database. To initialize the computer, a WH transformation is performed on each qubit individually, thereby preparing the overall state into an equal superposition of each of the database elements, e.g., $(|00\rangle+|01\rangle+|10\rangle+|11\rangle) / 2$. Next, an 'Oracle" simultaneously examines all database elements, and marks one (or more) of them with a $\pi$ phase-shift. In general, the Oracle might perform some computation on each element (e.g., test whether it is prime), and mark only that element which yielded a certain result (e.g., "prime"). For our proof-of-principle demonstration, the Oracle merely marks one element of the database (e.g., the state $|01\rangle$ if the second element is the desired one), while leaving the others unchanged. Viewed as a computation, our Oracle accepts a user-specified input (e.g., '2'), and marks which database element matches that number.

Finally, a series of transformations accomplish the 'inversion about the mean' operation at the heart of the amplitude-enhancement technique introduced by Grover 22. These are: (1) apply a second WH transformation to each bit; (2) apply a $\pi$ phase-shift to all but the first element $(|00\rangle)$ of the database; and (3) again apply a final WH transform to each bit. The net result of these

\footnotetext{
${ }^{1}$ The BS transformations are well-known: $a \rightarrow(a+i b) / \sqrt{2}$; $b \rightarrow(i a+b) / \sqrt{2}$. A $-\pi / 2$ phase shift in the $b$ mode before and after the BS yields the WH transform 10.
} 
last three operations is to transfer some of the amplitude from the non-special elements to the marked one. Remarkably, for $N=4$ (i.e., a 2-bit database), all of the amplitude is transferred to the desired element in a single run of the quantum circuit. In general for larger $N$, running the circuit $\sim \pi \sqrt{N} / 8$ times will lead to a final state in which the magnitude of the marked element's amplitude is greater than $1 / \sqrt{2}$ - a measurement on the system will then yield this element more than half the time [14]. For our experiment we focused on the simplest case of a 2-bit, 4-element database. Below we discuss the extension to more bits.

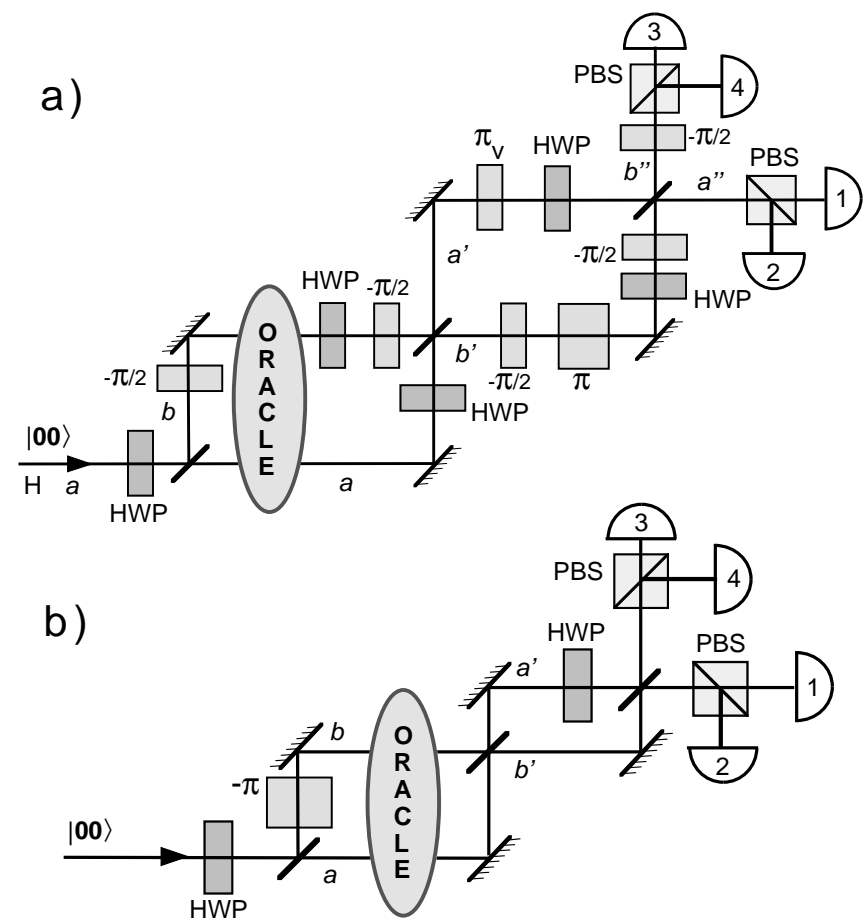

FIG. 1. Grover's technique for efficiently searching a database. (a) A one-to-one optical implementation of the circuit, for the case of $n=2$ bits (one represented by polarization and the other by spatial mode.) All half waveplates (HWP) are oriented at $22.5^{\circ}$. (b) A 'compiled' version of the algorithm, where many of the components have been consolidated. The first and second HWPs are at $22.5^{\circ}$ and $45^{\circ}$, respectively.

\subsection{Optical Coding}

Figure 1a shows the optical layout corresponding to a direct 1-to-1 implementation of Grover's algorithm, in which each operation is implemented independently. A HWP oriented at $22.5^{\circ}$ performs the initial WH transformation on the polarization bit, while the WH transformation on the spatial-mode bit is performed by the first 50-50 BS and the $-\pi / 2$ phase shifter in the reflected path. The oval represents the Oracle. Below we will discuss a practical implementation of this device; for the moment consider simply inserting a waveplate in the spatial path $a$ or $b$, which depending on its orientation gives a $\pi$ phase shift to either $\mathrm{H}$ or V-polarization - the Oracle can thus flip the sign of any one of the database elements $|a H\rangle,|a V\rangle,|b H\rangle$, or $|b V\rangle$. For example, if the second element is the marked one, the state of the system after the Oracle is given by $(|a H\rangle-|a V\rangle+|b H\rangle+|b V\rangle) / 2 \equiv$ $(|00\rangle-|01\rangle+|10\rangle+|11\rangle) / 2$. Note that this is an entangled state of the two qubits - it cannot be factorized into a product of a term involving only the polarization and a term involving only the spatial path.

The first WH transformation of the 'inversion-aboutthe-mean' subroutine on the polarization bit is again accomplished by HWP's, and on the spatial mode by combining paths $a$ and $b$ on a second 50-50 BS (with associated $-\pi / 2$ phase shifters). The minus sign on all but the $|00\rangle\left(\equiv\left|a^{\prime} H\right\rangle\right)$ element is obtained by a $\pi$-phase shift thickness of non-birefringent glass in path $b^{\prime}$ (or by simply lengthening path $b^{\prime}$ by $\lambda / 2$ ), and a half waveplate in path $a^{\prime}$, oriented with its fast axis horizontal (and labelled ' $\pi_{V}$ ' in figure 1a). The final WH transformations are again produced with HWP's and a 50-50 BS. We examine each of the outputs with a polarizing beamsplitter (PBS) in the $\mathrm{H}-\mathrm{V}$ basis.

\section{3. 'Compiling'}

This basic optical circuit for Grover's algorithm may be simplified considerably by consolidating some of the optical transformations. For example, because we are free to choose the order in which the WH transforms are applied to the polarization and spatial modes (i.e., these operations on different qubits commute), we may move the HWP's in paths $a$ and $b$ after the second BS, and combine them with elements in the second interferometer the HWP- $\pi_{V}$-HWP combination in path $a^{\prime}$ is equivalent to a single HWP oriented along $45^{\circ}$, and the HWP-HWP combination in path $b^{\prime}$ yields the Identity transformation, i.e., no element at all. After a few other consolidations of various phase shifts, the optical circuit of figure 1a may be simplified to that in figure $1 \mathrm{~b}$.

To understand the device performance, we calculate, for example, the probability that a photon will exit to detector 1 , by summing the amplitudes of the four possible paths, and taking the absolute square:

$$
\begin{aligned}
\mathrm{P}(1) & =\mid \frac{1}{\sqrt{2}} \frac{1}{\sqrt{2}} A_{a V} \frac{1}{\sqrt{2}} \frac{1}{\sqrt{2}}+\frac{1}{\sqrt{2}} \frac{i}{\sqrt{2}} A_{b V}(-1) \frac{i}{\sqrt{2}} \frac{1}{\sqrt{2}} \\
& +\frac{i}{\sqrt{2}} \frac{i}{\sqrt{2}} A_{a H} \frac{1}{\sqrt{2}} \frac{1}{\sqrt{2}}+\left.\frac{i}{\sqrt{2}} \frac{1}{\sqrt{2}} A_{b H}(-1) \frac{i}{\sqrt{2}} \frac{1}{\sqrt{2}}\right|^{2} \\
& =\frac{1}{4}\left|A_{a V}+A_{b V}-A_{a H}+A_{b H}\right|^{2}
\end{aligned}
$$

where the $A$ 's represent the action of the Oracle, and each path's amplitude is calculated starting at the output (i.e., the first factor in each term represents transmission or reflection at the last non-polarizing beamsplitter). The 
Oracle adds a relative phase of $\pi$ to the amplitude associated with the marked element of the database, while leaving the others unchanged. We see that for the condition $\left\{A_{a H}=-1 ; A_{a V}=A_{b H}=A_{b V}=1\right\}, \mathrm{P}(1)=1$; otherwise, if any of the other amplitudes received the $\pi$ phase shift instead, $\mathrm{P}(1)=0$. Thus, a click at detector 1 unambiguously determines that the marked database element was $|00\rangle$. Similarly, one can show that $\mathrm{P}(2)=1$ for $A_{a V}=-1$, $\mathrm{P}(3)=1$ for $A_{b H}=-1$, and $\mathrm{P}(4)=1$ for $A_{b V}=-1$.

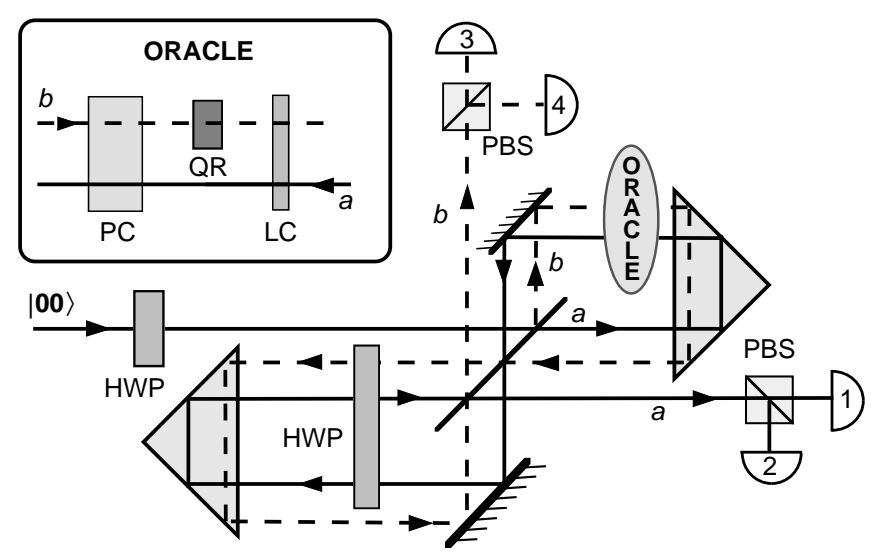

FIG. 2. Schematic of the self-stabilized optical setup used to implement Grover's algorithm. Inset: Oracle.

\section{Experimental Realization}

\subsection{Stabilization and the 'Oracle'}

One experimental constraint in any practical implementation is that the interferometer path lengths must be kept equal modulo $2 \pi$ to have the desired transformation. Instead of incorporating active stabilization, we chose to use a more robust interferometer geometry, based on displaced Sagnac interferometers. In the usual Sagnac interferometer, the interfering paths arise from light propagating clockwise $(\mathrm{CW})$ and counter-clockwise (CCW) through the system; because the optical path lengths are identical for both directions, the interferometer is automatically stabilized. The disadvantage is that one cannot independently address the CW and CCW modes any optical element in one mode is automatically in the second as well. Using a displaced geometry we can maintain the stability advantages of the usual Sagnac design, along with the ability to independently affect the CW and CCW modes. The resulting experimental setup is shown in figure 2 月.

\footnotetext{
${ }^{2}$ In actuality, the HWP (oriented at $45^{\circ}$ ) in the second interferometer acts on three of the paths instead of just one, ensuring that a photon horizontally-polarized in one of the
}

The inset to figure 2 shows one implementation of the Oracle, which requires no moving parts 3 , and which was used to obtain the data presented here. It consists of a Pockels cell (PC) [Lasermetrics \#Q1059], which acts as a piece of glass when no voltage is applied, and as a HWP oriented with the fast axis horizontal (i.e., $\mathrm{H} \rightarrow \mathrm{H}$ and $\mathrm{V} \rightarrow-\mathrm{V}$ ) when a voltage of $3.9 \mathrm{kV}$ is applied; a liquid crystal phase retarder (LC) [Meadowlark \#LRC-200700 ], which acts as a piece of glass when a voltage of $5.6 \mathrm{~V}$ is applied, and as a HWP with horizontal fast axis when a voltage of $2.2 \mathrm{~V}$ is applied; and a quartz rotator, which rotates any linear polarization by $90^{\circ}$. Consider, for example, the transformations when the applied voltages are $0 \mathrm{kV}$ and $2.2 \mathrm{~V}$ :

$$
\begin{array}{r}
a H \rightarrow a H \rightarrow a H ; b H \rightarrow b H \rightarrow b V \rightarrow-b V \\
a V \rightarrow-a V \rightarrow-a V ; \quad b V \rightarrow b V \rightarrow-b H \rightarrow-b H .
\end{array}
$$

The input state $a H \equiv|00\rangle^{\prime}$ is the only one to acquire a net relative $\pi$ phase shift. Similarly, the other three combinations of voltages on the Oracle components each apply the phase shift to a different element of the database.

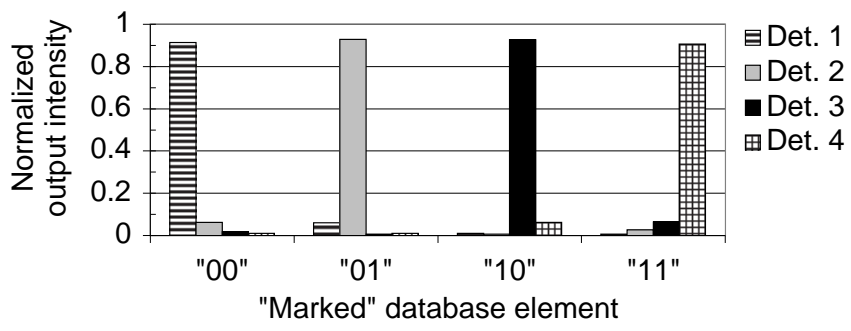

FIG. 3. Results obtained with the setup in figure 2 .

\subsection{Results and Extensions}

The system in figure 2 was tested by directing a horizontally-polarized beam from a 670-nm laser diode into the interferometer, and monitoring the output intensities for the four different settings of the Oracle. The results are shown in figure 3 . The circuit essentially performed as expected - the setting of the Oracle (i.e., the

retro-reflectors will be vertically-polarized in the other. Any birefringent phase-shift from the total internal reflections is thus the same for all four trajectories through the system, and factors out of the final result. This is a practical example of "bang-bang" quantum control 115. Also, we used the beamsplitter and mirrors at closer to normal incidence to reduce polarization-dependences.

${ }^{3}$ Oracles that rely on insertion or rotation of a waveplate would necessitate constant realignment of the interferometer, due to slight wedges and wavefront anisotropies typical in these elements. 
special element of the database) could be determined accurately with a single query $¥$. The average probability of error for a given port, as given by the relative output intensities, was less than $2.8 \%$, and is mostly due to slight wavefront distortions introduced by the Oracle elements.
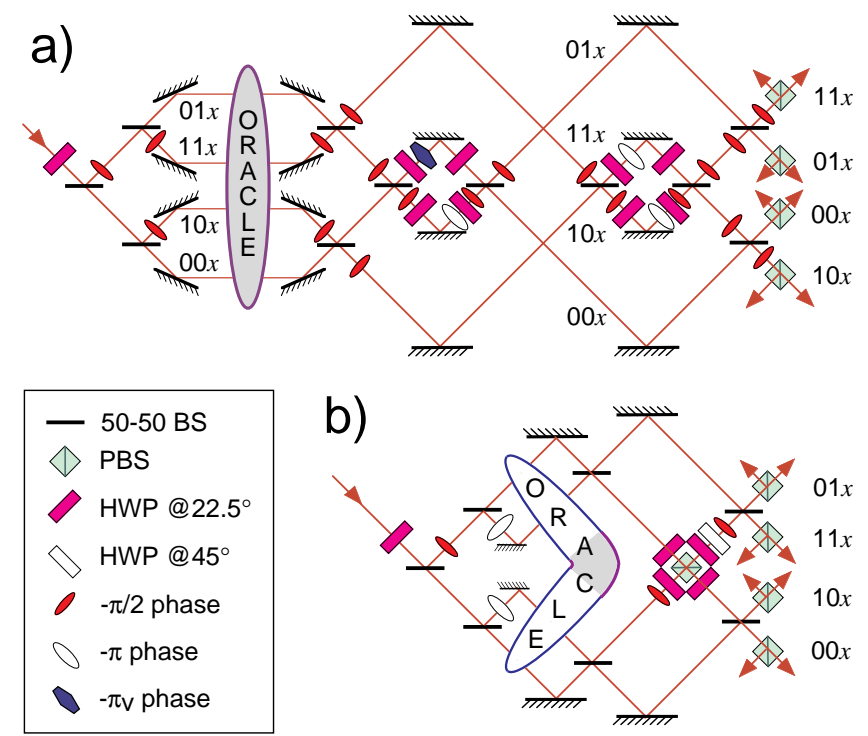

FIG. 4. Optical realization of Grover's algorithm for $n=3$ bits. A horizontally-polarized photon (polarization qubit $\equiv$ ' 0 '). The numbers represent the qubits of the spatial modes at various stages of the computation; the $x$ represents the value of the polarization qubit. (a) The uncompiled version. (b) A compiled implementation (but not yet consolidated to make multiple use of redundant components). A photon has a $25 / 32=0.78$ probability to exit via the port corresponding to the database element marked by the Oracle, and a 1/32 probability to exit via each of the other seven outputs.

It should be possible to extend the current work to a greater number of bits $n$ and a correspondingly larger database. One could use different frequencies of light, different orbital angular momentum modes [16], or even different photon occupation numbers. However, the most straightforward extension is simply to add extra spatial modes (via beamsplitters), so that, for example, whether the photon is transmitted at the first BS defines the first bit, whether it is transmitted at the next BS becomes the second bit, and so on. In figure $4 \mathrm{a}$ we show the direct optical coding of the Grover algorithm for a 3-qubit,

\footnotetext{
${ }^{4}$ While our experiment used many photons simultaneously for convenience, the identical results are predicted if single photons were used instead, i.e., only a single photon is needed to search the database. Our laser beam may be thought of as many noninteracting copies of identical quantum computers, just as each individual molecule in the bulk NMR schemes is like a separate 'computer', effectively isolated from the rest of the sample [阿.
}

8-element database, requiring 63 optical elements. One immediately sees the major limitation of these all-optical schemes, namely, that the number of optical elements grows as $\sim 2^{n}$. H. However, this may be mitigated to some extent by 'compiling' the algorithm (see figure 4b). We have shown that, in fact, the requisite number of elements may be reduced to 21 or less, by making redundant uses of some of the components. It is an open question how to achieve the maximal reduction, and to what extent reduction in an optical circuit implementation corresponds to reduction with other 'hardware'.

\section{Discussion}

It is natural to ask what features of quantum computation are embodied in our all-optical implementation of Grover's algorithm. In particular, we have seen that we can use different degrees of freedom to represent individual qubits, and that we can readily prepare superposition states. And although there are no CNOT gates in this particular algorithm, the conditional phase shifts applied in the amplitude-enhancement procedure, and by the Oracle itself, serve to entangle the different degrees of freedom. That one can so easily achieve such entanglement is one of the advantages of the all-optical implementations of quantum circuitry. It is related to the fact that the optical implementation is much easier for operations that transform a given state of the database (e.g., applying a conditional phase shift to one element), because each state corresponds to a different physical mode of the system. Conversely, operations that transform a given bit become very difficult, requiring $\sim 2^{n-1}$ optical components (e.g., to flip the polarization qubit requires a HWP in half of the paths). Contrast this with the standard multi-particle implementations of quantum computers, in which operations at the bit-level are more natural, while those on the overall states are among the most difficult.

There has been some confusion and controversy as to what extent a state such as that generated by the Oracle is truly entangled. On the one hand, unlike more traditional multi-particle entangled states, an entanglement in multiple degrees of freedom could not be used to demonstrate nonlocality, as in tests of Bell's inequalities. The reason, however, is not that the states could not violate a suitable Bell's inequality - they can 6; rather, it

\footnotetext{
${ }^{5}$ Curiously, implementations using bulk NMR have a similar limitation, because the signal to noise for $n$ spins (bits) drops as $1 / 2^{n}$, due to the decreasing likelihood that all bits (which are in a thermal distribution) start off in the ground state [3]. In contrast, our photons start off in a very pure state. Proposed schemes for ultracold NMR computers might also avoid this limitation 17.

${ }^{6}$ Because the polarization and spatial mode are commuting observables, they can both be determined simultaneously,
} 
is that there is no way to achieve the space-like separation of the entangled systems necessary to satisfy the underlying assumptions of Bell's inequalities. So, in this sense our entanglement is fundamentally different than multi-particle entanglement [11]. On the other hand, the multiple degree-of-freedom states do satisfy the usual theoretical criteria for entanglement [18]. For example, if we trace over one of the systems (for instance, the polarization), the other system is left in a completely mixed state. Experimentally, this means, e.g.,, that the final polarization analysis is required for the interference to be observed.

An interesting possibility for extending the capabilities of the present techniques is to incorporate the true entanglement [19] (and even hyper-entanglement in several degrees of freedom [20]) that exists between correlated photons produced via spontaneous parametric down-conversion (or, indeed, via other quantum computer embodiments). For instance, it was recently shown that one could distinguish all four polarization Bell states using only passive linear elements, if the photons were simultaneously entangled in energy or momentum 21.

Another major advantage of the all-optical methods is the virtual lack of decoherence, stemming from the fact that there are no preferred bases (e.g., the $\mathrm{H} / \mathrm{V}$ polarization basis is not preferred to $45 /-45)$. However, we can produce an adjustable decoherence simply by increasing the interferometer path-length imbalance relative to the coherence length of the light. This technique was recently used to prepare an arbitrary mixed state of polarization [22], and to investigate optically various schemes for quantum control of decoherence 15,23.

Finally, we note that by combining these all-optical techniques with the ideas of 'interaction-free measurements' 24,25, one can demonstrate a remarkable prediction by Jozsa that a quantum computer can yield an answer without ever actually running [26]! The simplest version is to insert our entire circuit (minus the PBS's and detectors) into one arm of a Mach-Zehnder interferometer, such that the mode from the empty arm of the MachZehnder is recombined with the ' $00 / 01$ ' spatial-mode output of our system. A single horizontally-polarized photon is input to the Mach-Zehnder, and the length of the empty arm is adjusted to give complete destructive interference for one output port of the recombining BS, when ' 00 ' is the marked element of the database (see figure $5 \mathrm{a}$ ).

If instead ' 01 ' is the marked element, a photon exiting our Grover circuit would be vertically-polarized, so no interference occurs at the recombining BS: a detector in the previously 'dark' output port now fires half the time. If the photon's polarization is vertical $(1 / 2$ of

the former with a simple polarizer, the latter with a (nonpolarizing) beamsplitter of arbitrary reflectivity. the time), we know the photon took the path containing the quantum computer (i.e., the Grover circuit 'ran') and that ' 01 ' was the marked element. However, 1/4 of the time, the photon exiting the 'dark' port will be horizontally-polarized (figure 5b). In this case, we know the marked element is not ' 00 ' (otherwise the destructive interference would have kept this port 'dark'), and that the photon absolutely did not take the interferometer path containing the Grover circuitry. If it had, either the photon would have vertical polarization (if the special element were ' 01 ') or the photon would have left the Grover system by its other output port (if the special element were ' 10 ' or ' 11 '). Hence, $1 / 4$ of the time we will have answered the question "Is the marked element of the database not '00'?" without ever running the computer. By incorporating high-efficiency schemes for interactionfree measurements [25], one can in principle arbitrarily decrease the probability that the Grover circuitry is actually run if the marked element is not ' 00 '. Note this is an intrinsically quantum mechanical effect, relying on the indivisibility of a single photon. Note also that although the photon may not have actually traversed the search algorithm optics, their alignment is still critical, and, for example, the electrooptic Oracle elements still require power.

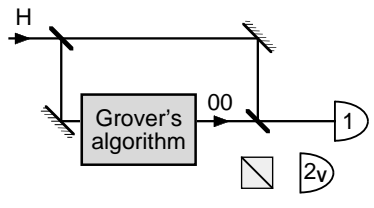

a)
(2H)

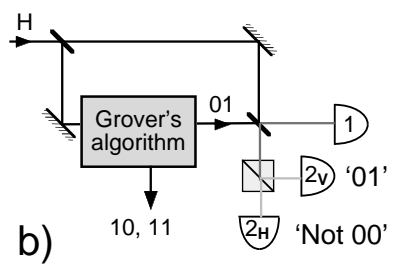

b)
FIG. 5. Incorporating 'interaction-free' measurements. (a) The interferometer is adjusted so that port 2 has complete destructive interference if the Oracle setting is ' 00 '. (b) If the Oracle setting is ' 01 ', then the exiting photon has vertical polarization, and no interference occurs - a detector in port 2 can now fire, indicating that the Oracle setting is not ' 00 '; half the time the photon in port 2 will nevertheless still have horizontal polarization, indicating that the quantum algorithm did not actually run.

In summary, we have demonstrated an all-optical realization of Grover's search algorithm. Because the qubits in our system are represented by different degrees of freedom, instead of separate quanta, many of the gate operations are much simpler to implement. We stress that our results are completely different than simply performing a (digital) calculation to predict the behavior - ours is a physical system that relies on superposition, interference, and non-factorizable states to function. Since these may also be classical phenomena, we conclude that many ingredients of quantum algorithms are not necessarily non-classical. The role of 'true' entanglement is to provide an exponential savings in resources. However, by 
compiling the optical circuity, algorithms involving several bits may be readily investigated.

\section{Acknowledgements}

We would like to acknowledge P. Wang and E. Waks for their assistance, and R. Jozsa for helpful discussions.

[1] Shor, P. W., 1994, in Proc. of the 35th Annual Symp. on Found. of Computer Science, edited by S. Goldwasser (IEEE Computer Society, Los Alamitos, CA), p. 116.

[2] Grover, L. K., 1997, Phys. Rev. Lett., 79, 325.

[3] Gershenfeld, N., and Chuang, I. L., 1997, Science, 275, 350; Cory, D. G., Fahmy, A. F., and Havel T. F., 1997, Proc. Natl. Acad. Sci. USA, 941634.

[4] Chuang, I. L., Gershenfeld, N., and Kubinuc, M., 1998 Phys. Rev. Lett., 80, 3408; Chuang, I. L., et al., 1998, Nature, 393, 143; Jones, J. A., Mosca, M., and Hansen, R. H., 1998, Nature, 393, 344.

[5] Monroe, C., et al., 1995, Phys. Rev. Lett., 75, 4714.

[6] Turchette, Q. A., et al., ibid., p. 4710.

[7] Reck, M., Zeilinger, A., Bernstein, H. J., and Bertani, P., 1994, Phys. Rev. Lett., 73, 58; Stenholm, S., 1996, Opt. Comm., 123287.

[8] Takeuchi, S., 1996, in Proc. of the 4th Workshop on Physics and Computation (PhysComp96), p. 299.

[9] Clauser, J. F., and Dowling, J. P., 1996, Phys. Rev. A, 53, 4587; Summhammer, J., 1997, Phys. Rev. A, 56, 4324.

[10] Cerf, N. J., Adami, C., and Kwiat, P. G., 1998, Phys. Rev. A, 57, R1477.

[11] Spreeuw, R. J. C., 1998, Found. Phys., 28, 361.

[12] Cleve, R., Ekert, A., Macchiavello, C. and Mosca, M., 1998, Proc. R. Soc. Lond., A454, 339; Ekert, A., 1998, Phys. Scripta, T76, 218.

[13] Farhi, E., and Gutmann, S., 1998, Phys. Rev. A, 57, 2403.

[14] Boyer, M., Brassard, G., Hoyer, P., and Tapp, A., 1998, Fortschr. Phys., 46, 493.

[15] Viola, L. and Lloyd, S., 1998, Phys. Rev. A, 58, 2733.

[16] Padgett, M. J. and Courtial, J., 1999, Opt. Lett., 24, 430.

[17] Kane, B., 1998, Nature, 393, 133.

[18] Bennett, C. H., DiVincenzo, D. P., Smolin, J. A., and Wootters, W. K., 1996, Phys. Rev. A, 54, 3824.

[19] Kwiat, P. G., et al., 1995, Phys. Rev. Lett., 75, 4337; Kwiat, P. G., et al., 1999, to appear as a Rapid Communication in Phys. Rev. A.

[20] Kwiat, P. G., 1997, J. Mod. Opt., 44, 2173.

[21] Kwiat, P. G., and Weinfurter, H., 1998, Phys. Rev. A, 58, R2623.

[22] Schwindt, P. D. D., Kwiat, P. G., and Englert, B.-G., 1998, submitted to Nature.

[23] Kwiat, P. G., and Berglund, A., 1998, unpublished.

[24] Eliztur, A., and Vaidman, L., 1993, Found. Phys., 23, 987.

[25] Kwiat, P. G., Weinfurter, H., Herzog, T., and Zeilinger,
A., 1995, Phys. Rev. Lett., 74, 4763.

[26] Jozsa, R., 1998, in Proc. of 1st NASA Int. Conf. on Quantum Computation and Quantum Communication, to appear in a special issue of Chaos, Solitons and Fractals. 